Article

\title{
Remembering Yury N. Gnedin at the Dawn of X-ray Polarimetry: Predictions of IXPE Observations of Neutron Stars
}

\author{
Jeremy Heyl (1)
}

check for

updates

Citation: Heyl, J. Remembering Yury

N. Gnedin at the Dawn of X-ray

Polarimetry: Predictions of IXPE

Observations of Neutron Stars.

Universe 2022, 8, 84. https://doi.org/

$10.3390 /$ universe 8020084

Academic Editors: Galina L. Klimchitskaya, Vladimir M. Mostepanenko and Nazar

R. Ikhsanov

Received: 31 December 2021

Accepted: 21 January 2022

Published: 28 January 2022

Publisher's Note: MDPI stays neutral with regard to jurisdictional claims in published maps and institutional affiliations.

Copyright: (C) 2022 by the authors. Licensee MDPI, Basel, Switzerland. This article is an open access article distributed under the terms and conditions of the Creative Commons Attribution (CC BY) license (https:// creativecommons.org/licenses/by/ $4.0 /)$.
Department of Physics and Astronomy, University of British Columbia, Vancouver, BC V6T 1Z1, Canada; heyl@phas.ubc.ca

\begin{abstract}
NASA's Imaging X-ray Polarimetry Explorer (IXPE) was launched in December 2021. It is 100 times more sensitive to polarized X-ray emission than any preceding mission and it is opening a new observational window into high-energy astrophysics. I outline Yury N. Gnedin's many contributions to understanding polarization from neutron stars and present new simulations of observations that IXPE will perform of the X-ray pulsar Hercules X-1 and the magnetar 4U 0141+561 in February 2022. These observations highlight and test particular models that Gnedin and collaborators first proposed. I outline how IXPE will provide unique constraints on the structure and kinematics of the boundary region between the accretion flow and the neutron star surface of Hercules X-1 and how IXPE will verify the predictions of vacuum birefringence for the magnetar $4 \mathrm{U} 0142+561$.
\end{abstract}

Keywords: magnetars stars; X-rays and stars; star atmospheres; polarization; plasmas; scattering; radiative transfer

\section{Introduction}

I had the privilege of working with Yury Nikolaesh Gnedin (1935-2018) during the summer of 1992 at the Pulkovo Observatory. That summer, several students from the United States were visiting Pulkovo, but I was the only one who spoke Russian, so I acted as cruise director; further, I conducted a research project with Yury Nikolaesh on the compactness problem in AGN and, more fortunately for me, I got to know him well doing that short time. Working with him was always fun as we tried to unlock mysteries and he also tried to make my visit rewarding beyond science, during what was a very trying time for Russia and Pulkovo. He arranged for me to visit the astronomical outpost overlooking Urtsalanj, Armenia (Figure 1). As our group was waiting on the tarmac at Mineralnye Vody Airport until well after midnight to fly over Georgia into Yerevan, supposedly to avoid anti-aircraft fire, I realized that, if Yury Nikolaesh had explained in detail what the trip would entail, I might have chosen not to do it and the story of the adventure that followed in Armenia itself would easily fill a different sort of article.

Knowing Yury Nikolaesh fueled a sense of adventure both scientifically and otherwise that has remained with me since, but, beyond that, the project that I conducted with him was my first one in high-energy astrophysics and, in particular, astrophysical manifestations of QED, which is a theme that my career has followed since. In fact, I find that, throughout my career, I have been retracing much of his work, more so than that of any of my other mentors. Now, as I write this nearly thirty years later, just a few weeks after the launch of NASA's Imaging X-ray Polarimetry Explorer (IXPE), we are waiting with keen anticipation the observations of so many phenomena in X-ray polarization, which Yury N. Gnedin began to outline more than fifty years ago. 


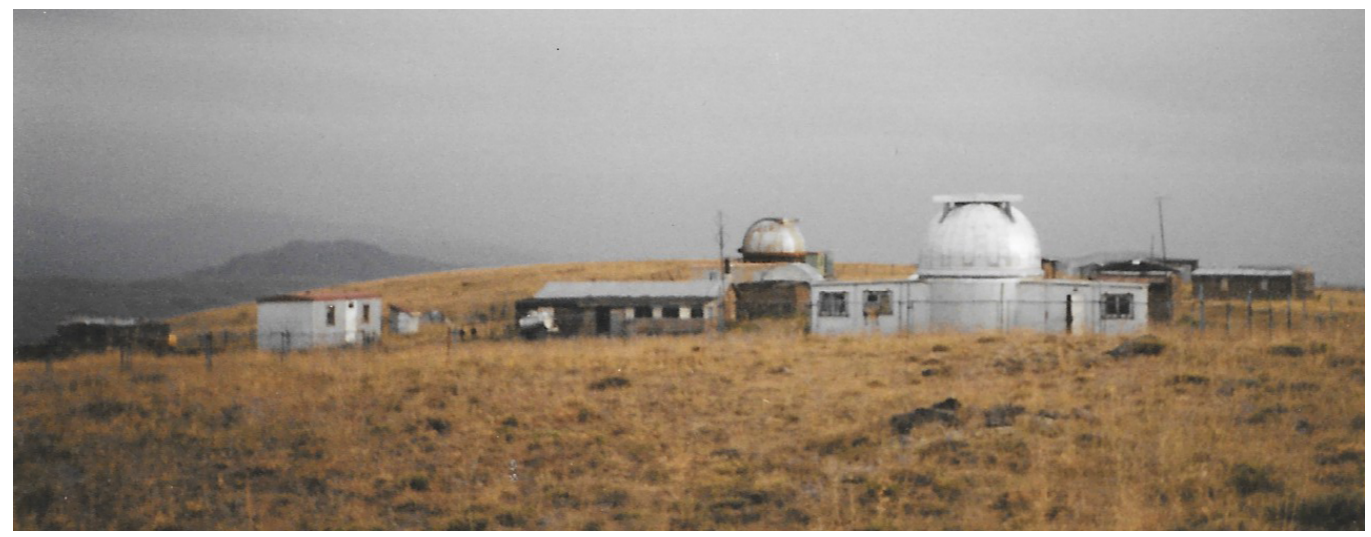

$$
\text { Thegun 10. H. }
$$

Figure 1. Top: wide view of the Ararat Scientific Site (taken by the author in August 1992). Bottom: inside cover of 150 Years of the Pulkovo Observatory, a souvenir from 1992.

\section{Polarized X-rays from Neutron Stars}

Although Yury N. Gnedin started by working on comets, e.g., [1-3], the discovery of neutron stars and the beginnings of high-energy astrophysics quickly dominated his early work. I here focus on the polarized X-ray emission from accreting and isolated neutron stars and how Gnedin's work informs our expectations even today. On 9 December 2021, the IXPE observatory was launched from Cape Canaveral, Florida. In February 2022, IXPE will be almost completely devoted to the study of two objects, the X-ray pulsar Hercules $\mathrm{X}-1$ and the magnetar $4 \mathrm{U} 0141+561$. Gnedin devoted significant studies to the first object, e.g., [4-6], and the implications of his studies, e.g., [7-11] of vacuum polarization play an important role in our understanding of the second object.

The IXPE observatory measures the polarization of X-rays in $2-8 \mathrm{keV}$ by measuring the direction of the initial photoelectron produced upon the absorption of an X-ray in a gas target. The total effective area of the three-mirror arrays is about $600 \mathrm{~cm}^{2}$. Once the quantum efficiency of the detector is included, the total effective area is decreased to about $75 \mathrm{~cm}^{2}$ at $2 \mathrm{keV}$ and $8 \mathrm{~cm}^{2}$ at $7 \mathrm{keV} \mathrm{[12].} \mathrm{The} \mathrm{interaction} \mathrm{between} \mathrm{an} \mathrm{X-ray} \mathrm{photon} \mathrm{and} \mathrm{the} \mathrm{electron}$ means that the direction of the photoelectron will be correlated with the polarization of the photon with $\operatorname{asin}^{2} \theta$ dependence. The magnitude of the correlation for fully linearly polarized radiation is known as the modulation factor and, for IXPE, it ranges from $15 \%$ at $2 \mathrm{keV}$ to $60 \%$ at $8 \mathrm{keV}$ [12]; therefore, although the effective area drops quickly with the increase in energy, the increase in the modulation factor mitigates this for the detection of polarization. IXPE is not sensitive to circular polarization. The energy resolution of the detectors is typically about $20 \%$, the angular resolution is about 20 arcseconds and the time resolution is one microsecond. All told, these specifications make IXPE a factor of 100 more capable than any X-ray polarimeter in space before it. The discovery potential is vast.

In the following sections, I describe what to expect from the first observations of X-ray polarization from Hercules X-1 and $4 \mathrm{U} 0142+561$ in the context of two particular models for the emission from these sources. Although I discuss some other models, this is not meant to be an exhaustive review but rather a review of how these expectations are connected with Yury N. Gnedin's work; consequently, much of the literature remains uncited. The models that I discuss have been presented elsewhere $[13,14]$, but the simulations for the planned 
February 2022 IXPE observations of 4U 0142+561 (1Ms) and Hercules X-1 (about 400 ks in total) are presented here for the first time.

\section{X-ray Pulsar Hercules X-1}

The discovery of the pulsating X-ray sources Centaurus X-3 [15] and Hercules X-1 [16] with the Uhuru satellite in the early 1970s uncovered a new type of celestial object, the accreting X-ray pulsar. Gnedin and Sunyaev [4] soon thereafter outlined the key features of a model of $X$-ray pulsars that hold to this day. The magnetic field channels the in-falling material onto the polar regions with a typical radius one-tenth that of the neutron star. The mildly relativistic material slows dramatically through a strong shock that radiates the gravitational energy away. They argued that the radiation came from a superposition of high gyrocyclotron resonances with a net spectrum that resembled that of thermal bremsstrahlung. Furthermore, they argued that the radiation would be polarized and beamed perpendicular to the magnetic field [17], a knife beam. The magnetic field of Hercules X-1 was measured through a cyclotron feature at about $60 \mathrm{keV} \mathrm{[18]} \mathrm{to} \mathrm{be} \mathrm{about}$ 100 times stronger than assumed by Gnedin and Sunyaev. Since the bulk of the emission lies below the cyclotron energy, the gyrocyclotron model of Gnedin and Sunyaev [4] does not hold. However, they argued, in a subsequent paper [17], that, if the bulk of the emission lies below the cyclotron resonance and the accretion shock lies close to the surface, the small opacity for photons traveling along the magnetic field results in a pencil beam along the field direction. This scenario is the basis for the slab models for X-ray polarization $[19,20]$.

Additionally, because the magnetic field is stronger than that assumed in [4], the accretion flow is channeled into a much smaller spot (about $0.1 \mathrm{~km}$ vs. $1 \mathrm{~km}$ ) and, for sufficiently high accretion rates, the flow piles up onto the surface in an accretion column (see Figure 2), where the accretion shock may lie well above the surface. In this situation, modern models for Hercules X-1, such as that of Becker and Wolff [21], find that the photon production is dominated by magnetic bremsstrahlung and photons scatter many times, gaining energy from the electron flow and ultimately emerge from the walls of the column, i.e., a knife beam (with polarization modeled for a static column in [22,23]). These models generally predicted a polarized fraction of about ten percent for the pencil beam and up to fifty percent for the knife beam in the range of $2-8 \mathrm{keV}$.

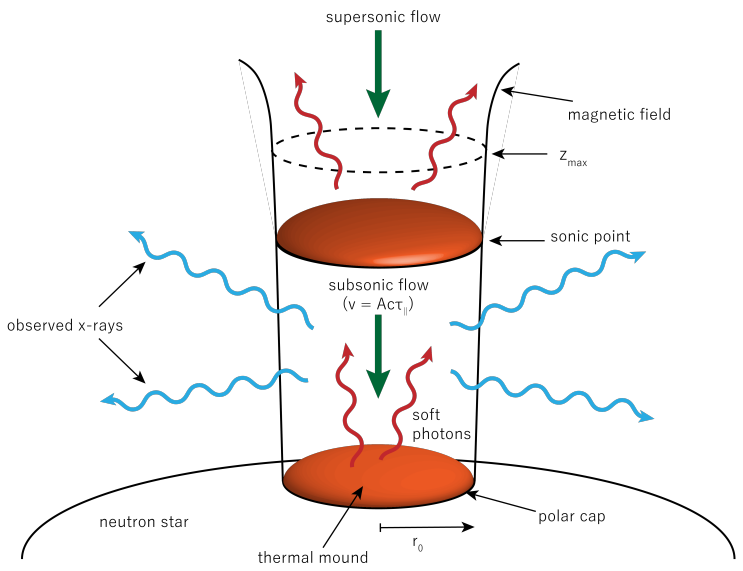

Figure 2. Schematic of the accretion flow near the surface of Her X-1; adapted from [21,24].

Although these models did not fit the broadband spectral energy distribution of X-ray pulsars such as Hercules X-1, it was nearly thirty-five years before new models for the polarization were developed $[13,24]$. Caiazzo and Heyl followed the spirit of Gnedin and Sunyaev [6,17] and focused on the scattering process itself to understand both the polarization and directional pattern of the emerging radiation. They used a matrix formalism [25] to find the asymptotic polarization state and radiation pattern within the plasma after many scatterings and study the final scattering before escape to derive the final 
polarization and emission pattern. To determine the final observed polarization and pulse profile, they included gravitational lensing, Doppler beaming and vacuum birefringence.

The results of two models that account for the observed pulse profile and cyclotron line of Hercules X-1 are depicted in Figure 3. The geometry of the two models is quite different. The model in the left-hand panels consists of emission from a single accretion column. The angle between the accretion column and the rotation axis of the neutron star is 86 degrees and the angle between the rotation axis and the line of sight is 83 degrees. It is nearly an orthogonal rotator and the bulk of the emission comes when the column is pointing away from the observer. Because the column is tall (seven kilometers) and the emission is beamed toward the surface, gravitational lensing dramatically amplifies emission in this configuration. The right-hand panels depict a geometry with two accretion columns. The columns make an angle of 42 degrees with respect to the rotation axis and the line of sight makes an angle of 50 degrees with respect to the line of sight. Again, the pulse is centered at the time when one of the accretion columns is pointing nearly directly away from the observer. The relativistic effects turn the typical rules for knife and pencil beams around. Pencil beams typically exhibit a minimum in the polarized fraction in the pulse accompanied with a rapid change in the polarization angle; on the other hand, a knife beam typically exhibits a peak in the polarized fraction and a slow change in the polarization angle during the pulse, e.g., [26]. The models presented in Figure 3 show a combination of these features. The polarized fraction reaches a peak in the pulse as for a non-relativistic knife beam, but the rate of change in the polarization angle is rapid through the pulse as in a pencil beam.

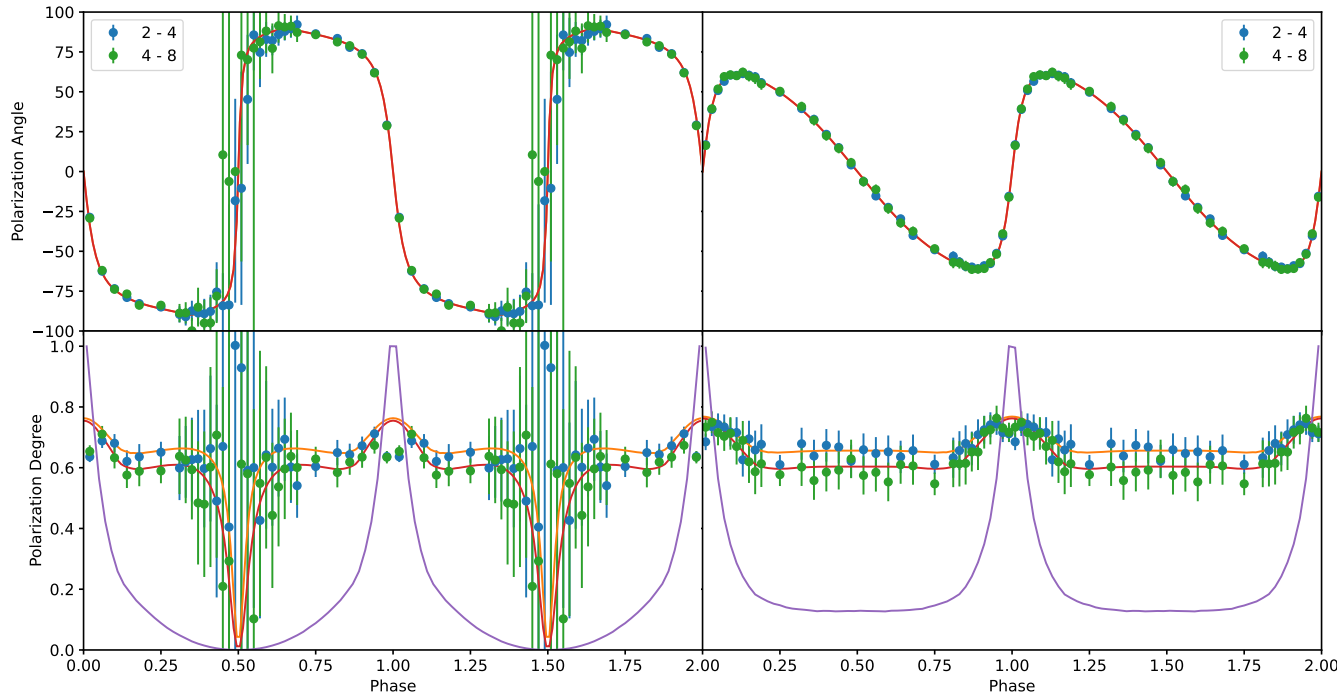

Figure 3. Predicted polarization of Her X-1 from the models of [13] with a $70 \mathrm{ks}$ observation with IXPE. The purple curves in the lower panels depict the total counts from the accretion column in the model. The left panels show the result with a single accretion column and the right panels depict two accretion columns. The large error bars near phase 0.5 in the left panel result from the lack of flux from the accretion column at this phase. The slight discrepancy near phase 1.0 between the measured and model polarization fraction results from smearing the polarization direction over the bin and can be avoided with an unbinned analysis.

Figure 3 also depicts the anticipated observations and uncertainties for a $70 \mathrm{ks}$ observation of Hercules X-1. In fact, IXPE will observe Hercules X-1 for a total of about $400 \mathrm{ks}$ in February 2022 over six visits to span its 35-day precession period. The simulation depicts the results for a single visit. The instrument will be able to determine whether the relativistic model [13] accounts for the observations. It will be also able to verify the Becker and Wolff picture [21]. The results of the ixpeobssim simulation [27] are for a phase-binned calculation, 
so the rapid variation in the polarization angle through the pulse reduces the observed polarization in the center of the pulse for both geometries. However, because IXPE tags the photon arrivals with an accuracy of 1 microsecond, it will be straightforward to determine the polarization fraction reliably, even when the angle changes rapidly. The combination of a rapidly varying angle and a maximum in both the count rate and the polarization fraction is the unique signature of the model. A static accretion slab or column yields different signatures, as discussed above.

\section{The Magnetar $4 \mathrm{U} 0142+561$}

Although Yury N. Gnedin only wrote a single paper about a magnetar per se [28], his work with George Pavlov, among others, outlined many of the crucial physical processes at play for the production and propagation of radiation for magnetars. In particular, he examined the interactions between photons and atoms in strong magnetic fields and highlighted that, even at the high temperatures of neutron star atmospheres, the material may have a significant neutral fraction [29]. Both this and the discussions on scattering in strong magnetic fields [6], when combined with the equations of radiative transfer in a strong magnetic field [7], form the basis for calculations of neutron star atmospheres, e.g., [30-34].

Additionally, Yury N. Gnedin and colleagues outlined the importance of vacuum birefringence from QED in the observations of neutron stars, e.g., [8-10], and, in particular, the spectral features that it can produce [11]. Following the spirit of the paper so far, the focus is X-ray polarization. Most important, for our discussion, is the argument of Novick et al. [35] whereby vacuum birefringence would generate a large phase difference between the normal modes and significant depolarization. Novick et al. wrote, "We conclude that it is extremely unlikely that polarized X-ray emission which arose from the surface of a magnetic neutron star would be observed". Gnedin et al. [9] countered the argument of Novick et al. by pointing out that a large phase retardation between the normal modes is a necessary but not sufficient condition for depolarization and, in fact, if the emission consists of an incoherent superposition of normal waves, no depolarization occurs, unless the properties of the birefringence vary too quickly. Birefringence would preserve the polarization along the path of the radiation. Furthermore, later work demonstrated that vacuum birefringence causes the direction of polarization in the X-rays for typical neutron stars to follow the direction of the magnetic field (the radiation remains in one of the normal modes) until a distance far from the surface of the neutron star, the polarizationlimiting radius [36,37]. In particular, this QED effect makes the observed polarization many times larger than one would expect from emission coming from a large portion of the neutron star surface [38]. The result of this effect is shown in Figure 4. The left-hand panel shows the polarization map at the surface of the star, in particular, the direction of the polarization of the extraordinary mode. Across the stellar surface, the direction varies significantly, so, if one were to sum the total polarized radiation in this case, one would obtain a small polarized fraction. The right panel depicts the situation with vacuum birefringence. At a large distance from the surface of the star, the magnetic field follows a dipole pattern; its projection in the plane of the sky would be parallel to the magnetic axis, in this case, horizontal. The polarization vectors that still follow the extraordinary mode are all vertical and the total polarization integrated over the surface of the star can be large if the polarization fraction at emission is large.

Caiazzo et al. [14] presented a comprehensive suite of models to account for the broadband emission from the magnetar 4U 0142+561 [39]. In all cases, they assumed that vacuum birefringence existed at the level predicted by QED. Figure 5 shows the results for a particular model that assumes that all of the emission from 2 to $8 \mathrm{keV}$ originates from a fully ionized hydrogen atmosphere [30], where the temperature and magnetic field follow a dipole distribution [40] with an additional polar hot spot. The figure shows a simulation of the planned 1 Ms IXPE observations for February 2022. In principle, other processes may contribute to this energy range, especially resonant cyclotron scattering [41-43]; however, 
for clarity, these are neglected here. With vacuum birefringence, the observed polarization fraction is high (nearly 100\%) over the entire energy band. On the other hand, without vacuum birefringence, the observed polarized fraction is low at the low end of the IXPE band when the emission originates over the entire surface, so, from the left panel of Figure 4 , the integrated polarization is small. At the high end of the IXPE band, the radiation comes from a very small portion of the stellar surface (the polar hot spot). Over this small region, the magnetic field in the plane of the sky is aligned in the horizontal direction; therefore, the resulting polarization is nearly vertical (i.e., in the extraordinary mode).
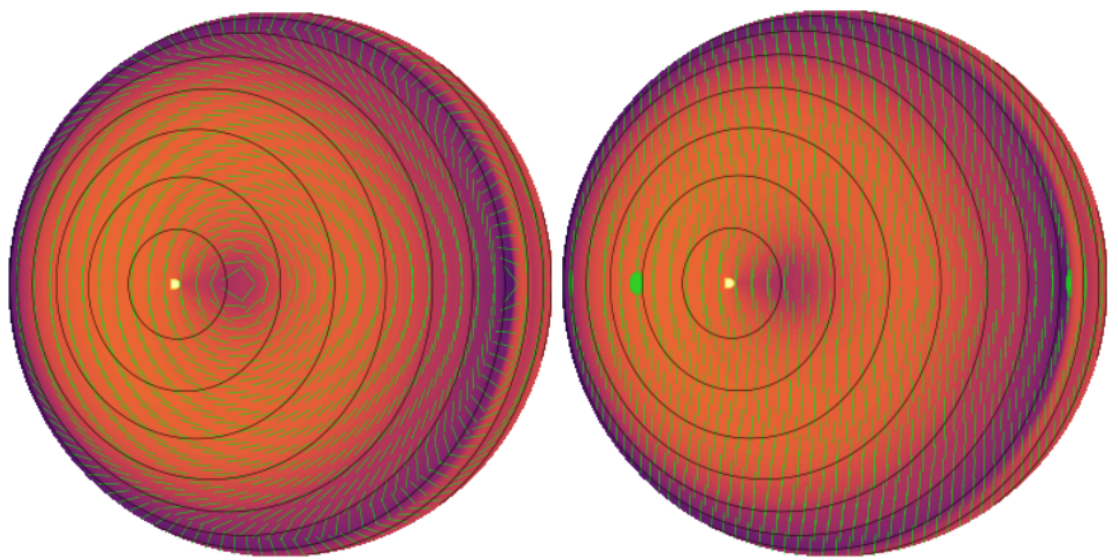

Figure 4. Surface polarization and emission map for the atmospheric model of $4 \mathrm{U} 0142+561$; from [14].

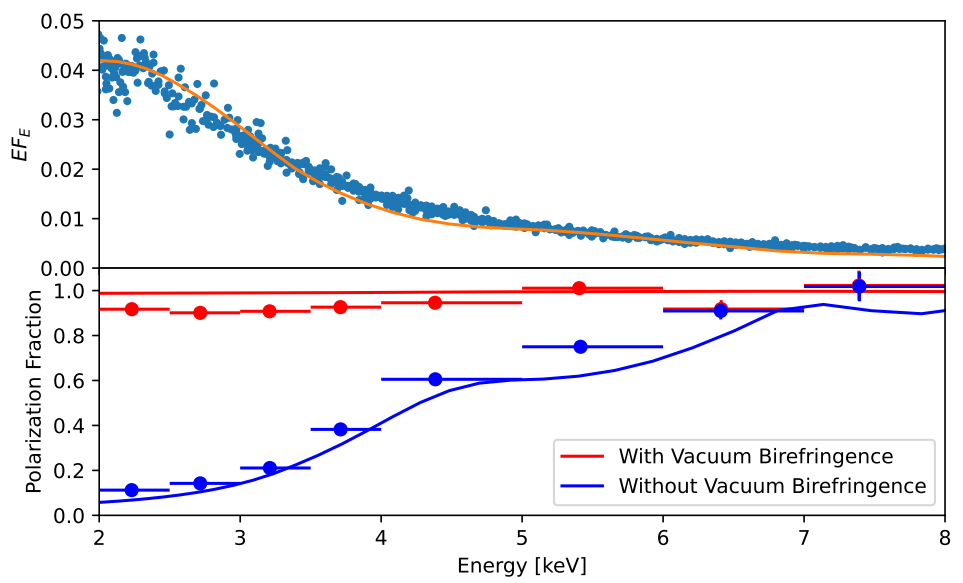

Figure 5. Predicted mean phase-resolved polarization of 4 U 0142+61 and phase-averaged flux [39] from the atmospheric models of [14] with a simulated 1 Ms observation with IXPE.

If the emission in the low end of the IXPE band originates in a gaseous atmosphere, the effect of vacuum polarization will be dramatically verified by IXPE observations. On the other hand, if the surface is condensed, e.g., [44,45], the expected polarization will be small at the low end of the IXPE band [14,46]. However, at the high-energy end of the IXPE band, resonant cyclotron scattering begins to contribute, resulting in a larger polarization and another potential verification of vacuum birefringence.

\section{Discussion}

In February 2022, the observations of X-ray polarization from Hercules X-1 and $4 \mathrm{U}$ 0142+61 with the recent launched X-ray observatory IXPE will verify the models and predictions that Yury N. Gnedin made at the dawn of high-energy astrophysics. Beyond these predictions and models for particular sources, Yury N. Gnedin, with collaborators, developed the framework of radiative transfer in a strongly magnetized plasma where vacuum 
birefringence, incoherent scattering and absorption all play key roles that are crucial to understanding polarized X-ray emission, regardless of the source. The broad scientific goals of IXPE from AGN to axions dovetail with Yury N. Gnedin's many contributions to astrophysics over the years, e.g., [47,48]. As we venture into the undiscovered country of astrophysical X-ray polarization, let us remember the maps and tools that he gave us for the journey.

Funding: This research study was funded by NSERC Canada.

Data Availability Statement: Data are available upon request to the Author.

Acknowledgments: The calculations were performed on SciServer; the author would like to thank Ilaria Caiazzo and Denis González Caniulef for useful conversations.

Conflicts of Interest: The author declares no conflict of interest.

\section{References}

1. Gnedin, Y.N.; Dolginov, A.Z. Particle Distribution in a Comet Head. Sov. Astron. 1966, 10, 143.

2. Dolginov, A.Z.; Gnedin, Y.N. A theory of the atmosphere of a comet. Icarus 1966, 5, 64-74. [CrossRef]

3. Gnedin, Y.N. The Inverse Problem in the Theory of Cometary Forms. Sov. Astron. 1967, 11, 485.

4. Gnedin, Y.N.; Sunyaev, R.A. The Beaming of Radiation from an Accreting Magnetic Neutron Star and the X-ray Pulsars. Astron. Astrophys. 1973, 25, 233.

5. Gnedin, Y.N.; Sunyaev, R.A. Luminosity of thermal X-ray sources witha strong magnetic field. Mon. Not. R. Astron. Soc. 1973, 162, 53. [CrossRef]

6. Gnedin, Y.N.; Sunyaev, R.A. Scattering of radiation by thermal electrons in a magnetic field. Sov. J. Exp. Theor. Phys. 1974, 38, 51.

7. Gnedin, Y.N.; Pavlov, G.G. The transfer equations for normal waves and radiation polarization in an anisotropic medium. Sov. J. Exp. Theor. Phys. 1974, 38, 903-908.

8. Gnedin, Y.N.; Pavlov, G.G.; Shibanov, Y.A. Influence of polarization of vacuum in a magnetic field on the propagation of radiation in a plasma. Sov. J. Exp. Theor. Phys. Lett. 1978, 27, 305.

9. Gnedin, Y.N.; Pavlov, G.G.; Shibanov, Y.A. The effect of vacuum birefringence in a magnetic field on the polarization and beaming of X-ray pulsars. Sov. Astron. Lett. 1978, 4, 117-119.

10. Pavlov, G.G.; Gnedin, Y.N. Vacuum polarization by a magnetic field and its astrophysical appearances. In Peculiar Stars, Strong Magnetic Fields and Gamma-ray Astronomy; Syunyaev, R.A., Ed.; All-Union Institute of Scientific and Technical Information: Moscow, Russia, 1983; pp. 172-219.

11. Gnedin, I.N. Effect of vacuum polarization in a strong magnetic field and spectral features of X-ray source emission. In $I A U$ Colloq. 115: High Resolution X-ray Spectroscopy of Cosmic Plasmas; Gorenstein, P., Zombeck, M., Eds.; Cambridge University Press: Cambridge, UK, 1990; pp. 78-84.

12. Ratheesh, A.; Rubini, A.; Marscher, A.; Manfreda, A.; Marrocchesi, A.; Brez, A.; Di Marco, A.; Paggi, A.; Profeti, A.; Nuti, A.; et al. The Imaging X-ray Polarimetry Explorer (IXPE): Pre-Launch. arXiv 2021, arXiv:2112.01269.

13. Caiazzo, I.; Heyl, J. Polarization of accreting X-ray pulsars-II. Hercules X-1. Mon. Not. R. Astron. Soc. 2021, 501, 129-136. [CrossRef]

14. Caiazzo, I.; González-Caniulef, D.; Heyl, J.; Fernández, R. Probing magnetar emission mechanisms with spectropolarimetry. arXiv 2021, arXiv:2112.03401.

15. Giacconi, R.; Gursky, H.; Kellogg, E.; Schreier, E.; Tananbaum, H. Discovery of Periodic X-ray Pulsations in Centaurus X-3 from UHURU. Astrophys. J. 1971, 167, L67. [CrossRef]

16. Tananbaum, H.; Gursky, H.; Kellogg, E.M.; Levinson, R.; Schreier, E.; Giacconi, R. Discovery of a Periodic Pulsating Binary X-ray Source in Hercules from UHURU. Astrophys. J. 1972, 174, L143. [CrossRef]

17. Gnedin, I.N.; Sunyaev, R.A. Polarization of optical and X-radiation from compact thermal sources with magnetic field. Astron. Astrophys. 1974, 36, 379-394.

18. Truemper, J.; Pietsch, W.; Reppin, C.; Voges, W.; Staubert, R.; Kendziorra, E. Evidence for strong cyclotron line emission in the hard X-ray spectrum of Hercules X-1. Astrophys. J. 1978, 219, L105-L110. [CrossRef]

19. Mészáros, P.; Nagel, W. X-ray pulsar models. I-Angle-dependent cyclotron line formation and comptonization. Astrophys. J. 1985, 298, 147-160. [CrossRef]

20. Kii, T.; Hayakawa, S.; Nagase, F.; Ikegami, T.; Kawai, N. Anisotropic X-ray transfer in a strongly magnetized plasma of the X-ray pulsar 4U 1626-67. Publ. Astron. Soc. Jpn. 1986, 38, 751-774.

21. Becker, P.A.; Wolff, M.T. Thermal and Bulk Comptonization in Accretion-powered X-ray Pulsars. Astrophys. J. 2007, 654, 435-457. [CrossRef]

22. Nagel, W. Radiative Transfer in a Strongly Magnetized Plasma-Part Two-Effects of Comptonization. Astrophys. J. 1981, 251, 288. [CrossRef] 
23. Kii, T. X-ray polarizations from accreting strongly magnetized neutron stars-Case studies for the X-ray pulsars $4 \mathrm{U} 1626-67$ and Hercules X-1. Publ. Astron. Soc. Jpn. 1987, 39, 781-800.

24. Caiazzo, I.; Heyl, J. Polarization of accreting X-ray pulsars. I. A new model. Mon. Not. R. Astron. Soc. 2021, 501, 109-128. [CrossRef]

25. Chou, C.K. Stokes Parameters for Thomson Scattering in a Strong Magnetic Field. Astrophys. Space Sci. 1986, 121, 333-344. [CrossRef]

26. Meszaros, P.; Novick, R.; Szentgyorgyi, A.; Chanan, G.A.; Weisskopf, M.C. Astrophysical implications and observational prospects of X-ray polarimetry. Astrophys. J. 1988, 324, 1056-1067. [CrossRef]

27. Pesce-Rollins, M.; Lalla, N.D.; Omodei, N.; Baldini, L. An observation-simulation and analysis framework for the Imaging X-ray Polarimetry Explorer (IXPE). Nucl. Instrum. Methods Phys. Res. A 2019, 936, 224-226. [CrossRef]

28. Gnedin, Y.N.; Ipatov, A.V.; Piotrovich, M.Y.; Finkel'Shtein, A.M.; Kharinov, M.A. Radio emission of the magnetar SGR 1806-20: Evolution of the magnetic field in the region of the radio afterglow. Astron. Rep. 2007, 51, 863-868. [CrossRef]

29. Gnedin, Y.N.; Pavlov, G.G.; Tsygan, A.I. Photoeffect in strong magnetic fields and X-ray emission from neutron stars. Sov. J. Exp. Theor. Phys. 1974, 39, 201.

30. Lloyd, D.A. Model atmospheres and thermal spectra of magnetized neutron stars. arXiv 2003, arXiv:0303561.

31. Lloyd, D.A.; Perna, R.; Slane, P.; Nicastro, F.; Hernquist, L. A pulsar-atmosphere model for PSR 0656+14. arXiv 2003, arXiv:0306235.

32. Lloyd, D.A.; Hernquist, L.; Heyl, J.S. Optical and X-ray Properties of Cooling Neutron Stars. Astrophys. J. 2003, 593, 1024-1031. [CrossRef]

33. Özel, F. Surface Emission Properties of Strongly Magnetic Neutron Stars. Astrophys. J. 2001, 563, 276-288. [CrossRef]

34. Ho, W.C.G.; Lai, D. Atmospheres and spectra of strongly magnetized neutron stars. Mon. Not. R. Astron. Soc. 2001, 327, 1081-1096. [CrossRef]

35. Novick, R.; Weisskopf, M.C.; Angel, J.R.P.; Sutherland, P.G. The effect of vacuum birefringence on the polarization of X-ray binaries and pulsars. Astrophys. J. 1977, 215, L117-L120. [CrossRef]

36. Shaviv, N.J.; Heyl, J.S.; Lithwick, Y. Magnetic lensing near ultramagnetized neutron stars. Mon. Not. R. Astron. Soc. 1999, 306, 333-347. [CrossRef]

37. Heyl, J.S.; Shaviv, N.J. Polarization evolution in strong magnetic fields. Mon. Not. R. Astron. Soc. 2000, 311, 555-564. [CrossRef]

38. Heyl, J.S.; Shaviv, N.J. QED and the high polarization of the thermal radiation from neutron stars. Phys. Rev. D 2002, 66, 023002. [CrossRef]

39. Tendulkar, S.P.; Hascöet, R.; Yang, C.; Kaspi, V.M.; Beloborodov, A.M.; An, H.; Bachetti, M.; Boggs, S.E.; Christensen, F.E.; Craig, W.W.; et al. Phase-resolved NuSTAR and Swift-XRT Observations of Magnetar 4U 0142+61. Astrophys. J. 2015, 808, 32. [CrossRef]

40. Heyl, J.S.; Hernquist, L. Almost analytic models of ultramagnetized neutron star envelopes. Mon. Not. R. Astron. Soc. 1998, 300, 599-615. [CrossRef]

41. Nobili, L.; Turolla, R.; Zane, S. X-ray spectra from magnetar candidates-I. Monte Carlo simulations in the non-relativistic regime. Mon. Not. R. Astron. Soc. 2008, 386, 1527-1542. [CrossRef]

42. Fernández, R.; Thompson, C. Resonant Cyclotron Scattering in Three Dimensions and the Quiescent Nonthermal X-ray Emission of Magnetars. Astrophys. J. 2007, 660, 615-640. [CrossRef]

43. Fernández, R.; Davis, S.W. The X-ray Polarization Signature of Quiescent Magnetars: Effect of Magnetospheric Scattering and Vacuum Polarization. Astrophys. J. 2011, 730, 131. [CrossRef]

44. van Adelsberg, M.; Lai, D.; Potekhin, A.Y.; Arras, P. Radiation from Condensed Surface of Magnetic Neutron Stars. Astrophys. J. 2005, 628, 902-913. [CrossRef]

45. Medin, Z.; Lai, D. Condensed surfaces of magnetic neutron stars, thermal surface emission, and particle acceleration above pulsar polar caps. Mon. Not. R. Astron. Soc. 2007, 382, 1833-1852. [CrossRef]

46. González Caniulef, D.; Zane, S.; Taverna, R.; Turolla, R.; Wu, K. Polarized thermal emission from X-ray dim isolated neutron stars: the case of RX J1856.5-3754. Mon. Not. R. Astron. Soc. 2016, 459, 3585-3595. [CrossRef]

47. Piotrovich, M.Y.; Gnedin, Y.N.; Buliga, S.D.; Natsvlishvili, T.M. Wavelength dependence of polarization and physical mechanisms of magnetic field generation in accretion disks around supermassive black holes in active galactic nuclei. Astron. Lett. 2014, 40, 459-463. [CrossRef]

48. Gnedin, Y.N.; Piotrovich, M.Y. New results in searching for axions by astronomical methods. Int. J. Mod. Phys. A 2016, 31, 1641019. [CrossRef] 